\title{
LA DIPLOMACIA COERCITIVA COMO ESTRATEGIA FRERTE A COREA DEL NORTE
}

\section{Coercive Diplomacy as a strategy vis à vis north Korea}

\author{
Gracia Abad Quintanal \\ Profesora Agregada de Relaciones Internacionales. \\ Universidad Nebrija \\ E-mail: gabad@nebrija.es \\ @graciaabad
}

Autor

Un repaso a la literatura, cada vez más amplia, sobre el conflicto con Corea del Norte nos lleva fácilmente a la conclusión de que frente a dicho Estado se han utilizado todas las estrategias posibles, pero ninguna ha resultado efectiva para lograr la solución del conflicto. Pues bien, en las páginas que siguen se tratará de mostrar cuáles han sido las estrategias utilizadas y, en la medida de lo posible, por qué han fracasado para, seguidamente plantear qué hay de novedoso en la que se está desarrollando actualmente. Por último se analizarán cuáles pueden ser las cuestiones clave para el éxito de la aproximación actual y se valorará hasta qué punto es posible que resulte finalmente fructífera.

Corea del Norte; proliferación; nuclear; disuasión; diplomacia coercitiva.

North Korea; proliferation; nuclear; deterrence; coercive diplomacy.

Q Key words

A review of the state of art regarding the conflict with North Korea leads us to the conclusion that all the strategies have been developed already to deal with that state, but none of them has resulted efective. Thus, in the pages to follow, we will try to show the strategies which have been developed and to some extent, the reasons why they have failed. Besides we will explain which are the new elements in the strategy which is currently in place. Last but not least, we will analyse the main questions which might condition the success of the current strategy and the extent to which this strategy may be really effective. 


\section{Introducción}

Si hay un aspecto recurrente en la literatura -tanto en artículos científicos como en divulgación o en prensa- sobre el conflicto con Corea del Norte es cuál puede ser la estrategia más eficaz para solucionar el problema. Sin embargo, en la mayor parte de los casos la respuesta a esa pregunta, con más o menos matices, es siempre la misma: ninguna.

De hecho, frecuentemente los propios protagonistas del conflicto ${ }^{1}$ (Martos, 2002, p. 13) han mostrado su disposición a solucionarlo insistiendo al propio tiempo en la falta de interés en ese sentido de la otra parte (Elliot, 2017, p. 10).

En efecto, la impresión que generalmente se transmite es que estamos ante un conflicto que se ha tratado de gestionar tanto mediante el recurso a medidas coercitivas y a la disuasión, como mediante la negociación y el ofrecimiento de incentivos y que ninguna de las opciones ha resultado realmente exitosa. En ese sentido, cabe pensar, incluso, que todas esas estrategias fallidas para lo que sí han servido es para que Pyongyang ganara tiempo y siguiera avanzando en sus esfuerzos para completar un verdadero programa de armas nucleares, algo que a día de hoy parece un objetivo relativamente próximo.

Sin embargo, y aun cuando esos planteamientos puedan tener sentido, algunas preguntas parecen no tener aún una respuesta definitiva: ¿de verdad se han probado todas las estrategias? ¿Cuál es la actual? ¿La aceptación de una Corea del Norte nuclear es irremediable? ¿Qué implicaciones tiene para la evolución del conflicto y de su gestión la existencia, desde 2011, de un nuevo líder en Corea del Norte, Kim Jong Un? ¿Hasta qué punto es diferente Kim Jong Un de su padre, Kim Jong Il y de su abuelo, Kim Il Sung, fundador del régimen norcoreano?

En ese sentido, en las páginas que siguen trataremos de dar respuesta a esas y otras preguntas, tratando con ello de acercarnos a la comprensión de la situación actual en Corea del Norte.

En ese esfuerzo de comprensión creemos que es fundamental tener presentes dos aspectos:

a) En la gestión del conflicto norcoreano se han abandonado estrategias pasadas basadas exclusivamente en la negociación o la disuasión y se ha apostado por una estrategia más compleja de diplomacia coercitiva.

b) Los cambios en el liderazgo, el régimen y la realidad norcoreanos unidos a la evolución del programa nuclear de Corea del Norte convierten a esa estrategia en acertada y, posiblemente, exitosa.

Con vistas a ver ambas cuestiones más en detalle, analizaremos los distintos intentos de solución, tratando de determinar hasta qué punto pueden ser considerados fundamentalmente como estrategias de negociación o disuasión y considerando tanto al contexto en el que han surgido como a sus resultados. En segundo lugar, analizaremos la situación actual y la estrategia que parece estar diseñándose, explicando por qué puede ser considerada como una estrategia de diplomacia coercitiva y tratando de establecer qué elementos requeriría para tener éxito y en qué medida cabe esperar que concurran tales elementos. El trabajo se cerrará con un apartado de conclusiones.

l Podemos considerar con Otomar J. Bartos que cabe hablar de conflicto "in a situation in which actors use conflict behavior against each other to attain incompatible goals and/or to express hostility". 


\section{Los intentos de solución del conflicto: las estrategias utilizadas}

Hasta el momento han sido dos las principales estrategias desplegadas con el fin de tratar de alcanzar una solución al conflicto planteado por los programas de armas nucleares y misiles de la República Popular y Democrática de Corea: la negociación acompañada de incentivos y la disuasión.

En cuanto a la negociación y los incentivos que la han acompañado, tan solo ha servido para que Corea del Norte jugara al engaño, se beneficiara de ellos y ganara tiempo en su avance imparable hacia unos programas de armas nucleares y misiles cada vez más sofisticados, mientras la comunidad internacional, empezando por los propios Estados Unidos, se limitaba a esperar el colapso de la RPDC (Stanton, Lee, y Klingner, 2017, p. 66) sin hacer demasiado para propiciarlo. Esa y no otra es la lectura que cabe hacer de las experiencias de la KEDO (Korea Energy Development Organisation, en sus siglas en inglés) (Abad Quintanal, 2007, p. 61), las Conversaciones a Seis Bandas y el acuerdo de 2007 (Abad Quintanal, 2008, p. 129) o el acuerdo del Día del Salto de $2012^{2}$ (Ford, 2018, p. 141), compromisos que Corea del Norte, que en realidad veía en ellos movimientos iniciales y silenciosos, pero que formaban parte de una estrategia de dominación más amplia (Stanton, Lee, y Klingner, 2017, p. 68), siempre ha acabado violando (Baker, 2017).

En realidad, parece cada vez más claro que Pyongyang ve en sus programas nucleares una garantía de su seguridad (Choong, 2017, p. 2), pues no olvidemos que no son sino un elemento particularmente importante de la estrategia de military first que pusiera en marcha Kim Jong Il, que llegó a decir que lo militar era "el pilar del socialismo" (Byman y Lind, 2010, p. 63). Al propio tiempo, la capacidad nuclear es para Corea del Norte, el mejor activo -casi el únicode que dispone para acudir a una negociación desde una cierta posición de fuerza (Garrido, 2017, p. 79). En consecuencia, ningún incentivo bastará para convencerle de que se desarme nuclearmente si cree que con ello pone en peligro la seguridad nacional o la supervivencia del régimen (Minnich, 2017, p. 51; Alagappa, 2017).

En lo que respecta a la disuasión, tanto los Estados Unidos como sus aliados en el nordeste de Asia, Corea del Sur y Japón, se han esforzado en elevar el coste que supone para Corea del Norte continuar con sus programas, haciéndole ver que podría estar en peligro incluso la supervivencia del régimen (Stanton, Lee, y Klingner, 2017, p. 66). En este sentido, han combinado instrumentos como las sanciones de distinto tipo, -en la línea de la disuasión por negación (Mitchell, 2012, p. 1)- con otros, como los ejercicios militares o las amenazas vertidas desde la Casa Blanca, especialmente notorias durante la administración de George W. Bush y en el curso de la actual, más en sintonía con la disuasión por represalia.

El problema es que esta última estrategia, en consonancia con lo que plantean los miembros de la cuarta ola de estudios de disuasión, que señalan que esta no va a tener ya la importancia

2 Acuerdo alcanzado como resultado del último proceso de negociación relativamente estructurado mantenido con Corea del Norte, en este caso, durante la Administración Obama. De la mano de dicho acuerdo la República Popular Democrática de Corea se comprometió a aceptar visitas de los inspectores de la IAEA y aplicar una moratoria tanto al lanzamiento de misiles de largo alcance como a la realización de pruebas nucleares como a las actividades nucleares desarrolladas en el reactor de Yongbyon, mientras EE. UU. reafirmaba tanto su compromiso con la Declaración Conjunta de 2005, como la ausencia de intenciones hostiles hacia Corea del Norte y se comprometía a proporcionarle 240.000 toneladas de asistencia alimentaria. El acuerdo quedaría roto por los posteriores incumplimientos norcoreanos.
Hasta el momento han sido dos las principales estrategias desplegadas con el fin de tratar de alcanzar una solución al conflicto planteado por los programas de Corea del norte: la negociación acompañada de incentivos y la disuasión. 
que tuvo durante la Guerra Fría (Knopf, 2010, p. 4), si bien ha dañado la economía norcoreana y ha podido servir para evitar que Corea del Norte lanzara un ataque contra Estados Unidos o sus aliados (Campbell, 2017, p. 9), tampoco ha sido útil como instrumento para convencer al gobierno de Pyongyang de que optara por el abandono y desmantelamiento de sus programas de armas nucleares y misiles ${ }^{3}$. De hecho, la República Popular Democrática de Corea no solo no ha abandonado tales programas, sino que ha avanzado tanto en la proliferación vertical como horizontal ${ }^{4}$.

Así, en relación con la primera ha ido progresando de forma cada vez más acelerada en sus programas, contaría ya con unas sesenta armas nucleares repartidas por todo el país (Pearlstine, 2017, p. 98) y es hoy un Estado con capacidad para atacar a Japón y Corea del Sur con armas nucleares, y que podría tenerla pronto también para atacar Estados Unidos (Stanton, Lee, y Klingner, 2017, p. 65). En relación con la segunda, ha seguido transfiriendo know how a Estados como Libia, Irán, Siria o Pakistán (Abad Quintanal, 2012, p. 60) y, cuando menos, alimentando el temor de que pudiera hacer lo propio con actores no estatales, en lo que ha constituido, por otra parte, una importante fuente de ingresos para Pyongyang (Byman y Lind, 2010, p. 63).

Más allá de ello, la coerción, si ha servido para algo en términos de la consolidación del régimen norcoreano, es para reforzar el control del mismo sobre el Estado y la población norcoreanos (Byman y Lind, 2010, p. 45).

En consecuencia no podemos sino hablar de una falta de efectividad de la estrategia de disuasión que probablemente y, más allá de la percepción de Pyongyang-que también apuesta por la disuasión, nuclear en este caso, aunque no falten quienes duden de su efectividad (Wilson, 2008, p. 421)- de que es la seguridad tanto del Estado como del régimen lo que está en juego, hay que atribuir a la conjunción de dos factores principales: la falta de credibilidad del disuasor, el distinto análisis coste-beneficio del disuasor y el potencial agresor y la elevada tolerancia al riesgo de este último (Jordan, 2013).

En efecto, en lo que respecta a la credibilidad, si bien las capacidades militares con que cuenta Estados Unidos están fuera de toda duda tanto para Corea del Norte como para el resto de los Estados del sistema internacional, no está tan clara la voluntad política, la determinación de Washington de recurrir a la fuerza si fuera necesario.

Una circunstancia que viene a sumarse, además, a la de la elevada tolerancia al riesgo exhibida por Corea del Norte y sus líderes. En efecto, ya el padre del actual líder, Kim Jong Il, pero sobre todo el propio Kim Jong Un ha mostrado una gran tolerancia al riesgo -hasta el punto de parecer en muchos casos irracional-, probablemente sabedor de que una guerra tendría un coste elevadísimo para Estados Unidos - mayor si cabe que el de permitir una Corea del Norte con armas nucleares (Cossa, 2017b)-, aun cuando saldría vencedor de ella con casi total seguridad (Jordán, 2013, p. 193), pero también porque cuenta con una mentalidad diferente.

3 En realidad cabe constatar en general el descenso de la eficacia de las estrategias de disuasión desde el fin de la Guerra Fría.

4 Se entiende por proliferación horizontal la transferencia de precursores, tecnología y know how para la fabricación de un determinado tipo de armas (nucleares en este caso) a terceros (Estados o actores no estatales) que no cuentan con dicho tipo de armas hasta el momento; mientras que proliferación vertical hace referencia a la mejora e incremento de los sistemas de un determinado tipo de armas (nucleares en el caso que nos ocupa) con que cuenta un Estado que ya poseía armamento de esa clase.

\section{En lo que respecta} a la credibilidad no está tan clara la voluntad política, la determinación de Washington de recurrir a la fuerza si fuera necesario 
De hecho, algunos de los autores de la cuarta ola de investigación sobre disuasión como Colin Gray o Payne consideran que el problema de Estados Unidos con Estados como Corea del Norte es que no entiende sus reacciones, pues tiene valores muy diferentes de los estadounidenses (Knopf, 2010, p. 6).

\section{La situación actual: el recurso a la diplomacia coercitiva}

Esa falta de efectividad de las estrategias empleadas hasta el momento ha llevado a la situación más peligrosa, en términos nucleares, desde la Crisis de los Misiles, en buena medida como consecuencia de la importantísima aceleración de los programas nucleares y de misiles del Estado que nos ocupa -sin parangón en ningún otro caso (Elleman, 2017, p. 25)-, en particular desde la asunción del liderazgo por parte de Kim Jong Un (Albert, 2017) -de las seis pruebas nucleares realizadas por Corea del Norte, cuatro se han hecho desde que el actual líder llega al poder- quien, probablemente sabedor del cuestionamiento de que era objeto en el momento de su ascenso al poder, parece haber optado por apuntalar su legitimidad mediante el perfeccionamiento de los programas armamentísticos que pusieran en marcha Kim Il Sung y Kim Jong Il, la profundización de la represión y el incremento de la tensión con EE. UU. y sus aliados. De hecho, la consolidación de la capacidad de disuasión norcoreana es uno de los dos pilares (el otro es el desarrollo de la economía) estrategia de Byunjing para consolidar su estado y su régimen (Ford, 2018, p. 135).

Así, desde principios de 2016 la República Popular Democrática de Corea habría lanzado casi 40 misiles de diferentes alcances (Garrido, 2017, p. 79). Más en concreto, desde julio de 2017, habría lanzado ya varios que, aunque con toda probabilidad carecen de la precisión que Pyongyang desearía - en parte por contar con unos sistemas de reentrada aún por perfeccionar y unos sistemas de guiado deficientes (Ford, 2018, p. 141)-, podrían ser capaces de alcanzar territorio estadounidense (Pearlstine, 2017, p. 98; Minnich, 2017, p. 44).

Figura 1. Lanzamientos de misiles de Corea del norte

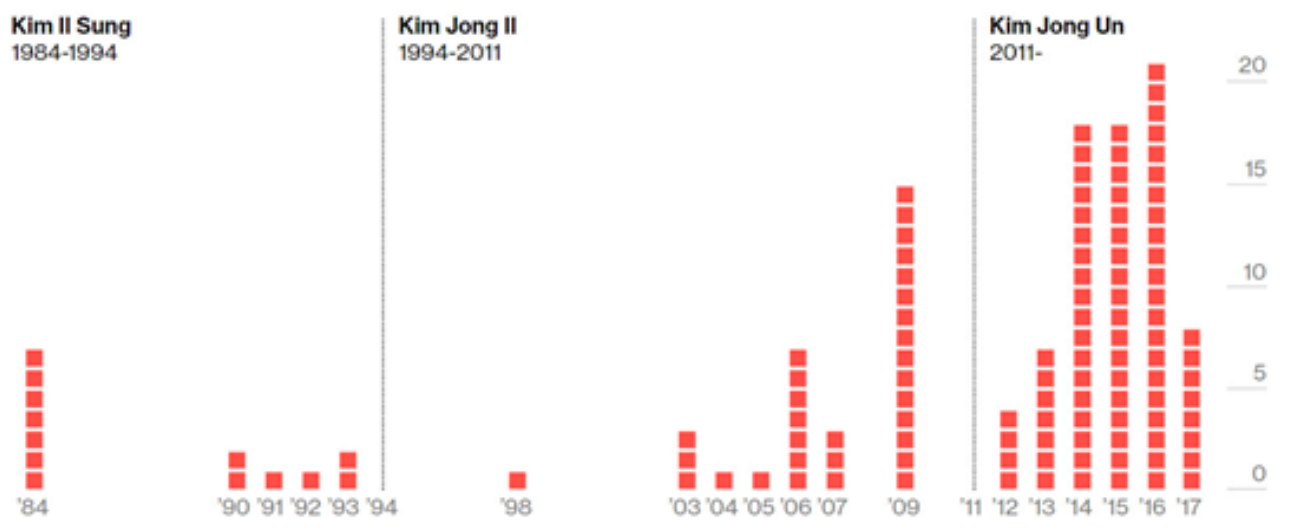

Esa falta de efectividad de las estrategias empleadas hasta el momento ha llevado a la situación más peligrosa en términos nucleares desde la Crisis de los Misiles 
A todo ello hay que sumar la realización, el 3 de septiembre de 2017 del sexto -y último hasta el momento- test nuclear de Corea del Norte que, a diferencia de los anteriores y, de acuerdo a algunos expertos sí podría ser ya una bomba de hidrógeno ${ }^{5}$.

Figura 2. Pruebas nucleares realizadas por Corea del norte

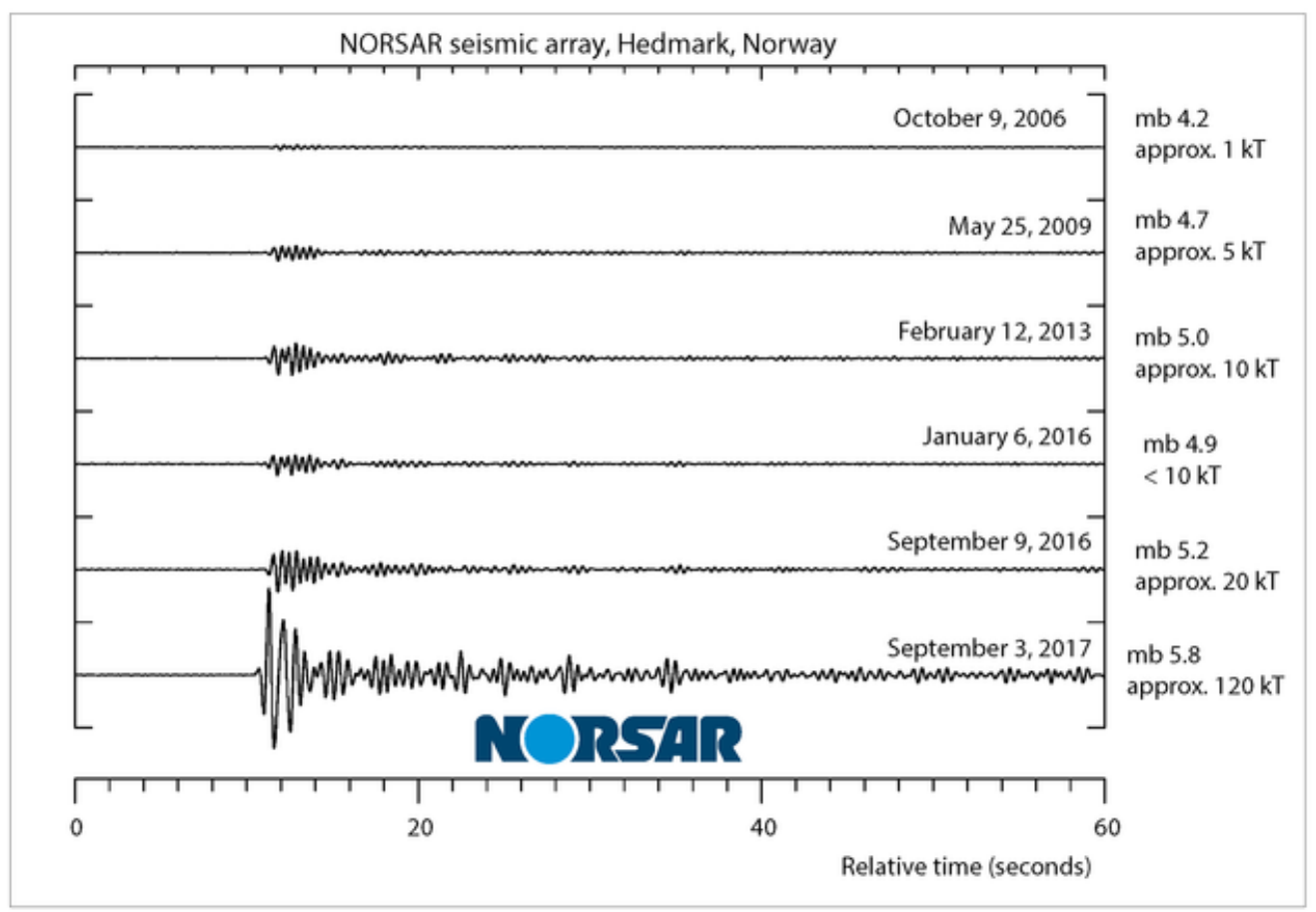

Fuente: Norsar

Esas circunstancias, sin ninguna duda, hacían necesario optar por una nueva estrategia que permitiera tratar de alcanzar mejores resultados que con las desplegadas anteriormente. Pues bien, un análisis cuidadoso de los acontecimientos de los últimos meses nos permite pensar que eso es precisamente lo que puede estar ocurriendo. En efecto, se habría empezado a reemplazar la estrategia de disuasión existente hasta hace poco por una, no de diálogo como tal, pues no se confía en ella y ya hemos visto cómo ha fallado reiteradamente en el pasado, sino de diplomacia coercitiva, entendida como "la explotación de la fuerza potencial para inducir al adversario a cumplir con las demandas que se le formulan" (Crystal, 2015, p. 3) y que, en el caso concreto que nos ocupa, supone el recurso a medidas de presión de distinto tipo para llevar a Corea del Norte a la mesa de diálogo. En otras palabras, se recurre a una estrategia que integra amenazas, uso de la fuerza limitado, persuasión, incentivos y adaptación en distintas proporciones (Levy, 2008, p. 539). Un cambio que no es extraño, pues es frecuente que se recurra a la diplomacia coercitiva cuando falla la disuasión (Jordán, 2013, p. 195).

Como apuntábamos, la adopción de esta estrategia supone, por una parte, mantener la presión y la amenaza de uso de la fuerza sobre Corea del Norte, pero, por otra, implica también ofrecer

5 Aunque Pyongyang afirmara que ya el quinto test había sido hecho con una bomba de hidrógeno. 
al gobierno de Pyongyang incentivos que le lleven a la negociación y a la conclusión de que la solución del conflicto no tiene por qué ser un juego de suma cero, sino que se puede llegar a una situación en la que todos ganen.

\subsection{La presión}

Si bien, sobre el papel, siempre ha habido sanciones y presiones a Corea del Norte, en la práctica su violación era mucho más común que su aplicación. Sin embargo, en este punto cabe constatar un antes y un después de la prueba nuclear efectuada por Pyongyang en enero de 2016. En este sentido, en términos de presión, y más allá de la retórica de la administración Trump que pasó de amenazar a Corea del Norte con "fuego y furia" a decirle que podrían "destruir totalmente Corea del Norte" (Ford, 2018, p. 142) y del refuerzo del aislamiento del régimen norcoreano a lo largo de los últimos meses (Baker, 2017), parece cada vez más claro que el elemento clave está siendo el estrangulamiento financiero a que, en buena medida a instancias de Washington, se está sometiendo a Corea del Norte. En efecto, Corea del Sur, que había mantenido abierto el complejo de Kaesong a través del cual Corea del Norte obtenía unos 100 millones de dólares al año sin que nadie controlara a que se destinaban, iba a optar por cerrarlo. Del mismo modo, incluso la República Popular China ha instado en los últimos tiempos a endurecer las sanciones contra la República Popular Democrática de Corea.

En esa misma línea se sitúa la aprobación, en febrero de 2017, por el congreso de los Estados Unidos de la North Korea Sanctions and Policy Enhancement Act, que venía a impedir que los bancos norcoreanos pudieran gestionar pagos en dólares. Partiendo de esa base, el Departamento del Tesoro ha congelado activos por valor de más de 200 millones de dólares a entidades de Corea del Norte (Stanton, Lee, y Klingner, 2017, p. 72).

Junto a ello EE. UU. ha instado a los principales Estados con relación con la República Popular Democrática de Corea a cesar en sus relaciones comerciales con ella, bajo la amenaza de impedirles el acceso al sistema dólar si no lo hacen. En ese mismo sentido está suspendiendo el acceso al sistema bancario estadounidense a aquellos bancos que faciliten transacciones relacionadas con el comercio con Corea del Norte (Cottee, 2017, p. 1).

La respuesta a tales planteamientos de un Estado clave en esta cuestión, como es la República Popular China -probablemente en parte como consecuencia de la estrategia de rendición de cuentas estratégica ${ }^{6}$ desplegada hacia ella por la Administración Trump (Choong, 2017, p. 2)-, no se hizo esperar, ya que desde principios de septiembre de 2017 ha pedido a los bancos chinos que respeten de forma estricta el régimen de sanciones impuesto a Corea del Norte.

Algo fundamental, ya que la presión podrá tener éxito en la medida en que haya consenso en su aplicación. Otra cuestión que también invita al optimismo es la aprobación de la Resolución 2375 a primeros de septiembre de 2017.

Junto a esas medidas de carácter económico y financiero, de cara a reforzar esa presión frente a Corea del Norte, es también importante la existencia en Washington de preparativos para ataques preventivos y un eventual cambio de régimen o estrategias encubiertas para minar el régimen propiciando un golpe de Estado o una insurrección (Ford, 2018, p. 142). Así, también

6 Continuadora de la estrategia de paciencia estratégica de la Administración Obama. 
los planes secretos de Estados Unidos y Corea del Sur en caso de un nuevo conflicto armado en la península coreana (OPLAN 5027 y OPLAN 5015), descubiertos supuestamente por un hacker norcoreano (Panda, 2017, p. 1), también podrían influir en esta dirección.

\subsection{El diálogo y los incentivos}

Por otra parte, se empiezan a fijar los términos que hagan posibles las conversaciones. En efecto, aprovechando la apertura al diálogo que se apreciaba en el discurso de año nuevo de Kim Jong Un (Jongsoo, 2018) - probablemente como consecuencia de la presión aplicada a Corea del Norte, pero también de haber alcanzado prácticamente sus objetivos en lo que respecta a sus programas nucleares y de misiles (Cossa, 2017a)- Estados Unidos y Corea del Sur han empezado a dar pasos en esa dirección. Así se ha abierto paso ya la celebración de una cumbre entre las dos Coreas a la que podría seguir otra entre Estados Unidos y Corea del Norte. Un formato que, en parte al menos, responde a las preferencias de Pyongyang, que siempre buscó la negociación bilateral con Washington y no formatos multilaterales como el de las conversaciones a seis bandas de la década pasada.

El desarrollo del proceso negociador si bien se puede condicionar al mantenimiento de la suspensión de nuevas pruebas, también se puede y debe incentivar con la suspensión (temporal al menos) de los ejercicios militares conjuntos celebrados habitualmente por Estados Unidos y sus aliados, en línea con lo que ya se hizo durante los pasados Juegos Olímpicos de Invierno, celebrados en la República de Corea (Jongsoo, 2018).

En el marco de dichas conversaciones se deberá hacer ver a Corea del Norte que el avance de la misma permitiría, progresivamente, la normalización de relaciones, la cooperación económica y comercial, la cooperación energética o la gestión de intereses en conflicto, cuestiones todas ellas que beneficiarían al Estado del nordeste de Asia (Minnich, 2017, p. 40).

Sobre esa base, el diálogo debería conducir, en el mejor de los casos, a la desnuclearización de Corea del Norte y, en el peor, al mantenimiento de sus programas nucleares "dentro" de la legalidad internacional y sin que amenacen la paz y seguridad internacionales. A tal efecto, sería necesario un triple compromiso norcoreano (Alagappa, 2017):

» De no utilizar armas nucleares contra Estados que no las poseen -incluidos algunos vecinos con los que no mantiene precisamente buenas relaciones como Japón o Corea del Sur-.

» De no transferir tecnología ni materiales para la fabricación de armas nucleares a Estados que no la poseen ni a actores no estatales.

» De proteger sus instalaciones nucleares de forma que no pueda haber accidentes y no puedan ser utilizadas por actores criminales.

\subsection{Las posibilidades de éxito}

Parece claro que las posibilidades de éxito de esta aproximación dependen en buena medida de que la combinación entre las medidas de presión y los incentivos sea la adecuada. En cuanto a las primeras, da la impresión de que, bien aplicadas podrían surtir efecto por cuanto una Corea del Norte absolutamente depauperada que, sin embargo, había empezado al 5\% bajo Kim Jong Un (Choong, 2017, p. 3) no querrá volver atrás, pero resta ver también si los incentivos se gestionan con igual habilidad.

\section{El diálogo debería} conducir, en

el mejor de

los casos, a la

desnuclearización

de Corea del norte

$y$, en el peor, al

mantenimiento de

sus programas

nucleares "dentro"

de la legalidad

internacional 
Ahora bien, como decíamos más arriba, nada ni nadie podrá convencer a Pyongyang de que se desnuclearice si, como ocurre actualmente, ve en su capacidad de disuasión nuclear la garantía de supervivencia del Estado y del régimen. En ese sentido, la negociación debe permitir convencer a Corea del Norte de que su seguridad no estará en riesgo en caso de que abandone los programas nucleares, sino más bien al contrario.

Una buena opción en este sentido, para la cual sería necesaria la cooperación de la República Popular China sería ofrecer como garantía de seguridad la disuasión extendida de esta última. Así, en la medida en que China esté dispuesta a ofrecerle a la República Popular Democrática de Corea garantías de que la defenderá en caso de un ataque estadounidense, Corea del Norte podría empezar a ver viable la opción del desarme nuclear (Bobbitt, 2017, p. 35).

Al propio tiempo, la presión ejercida debe acompañarse de ventajas económicas y de todo tipo lo suficientemente importantes para que Pyongyang, incluso si accede a abandonar sus programas nucleares, tenga la sensación de que también ha salido victorioso de la negociación (Cossa y Glosserman, 2017).

\section{Conclusiones}

En este sentido, cabe considerar que se equivocan quienes califican a la actual administración estadounidense por sancionar y dar incentivos de forma simultánea al régimen de Pyongyang: bien al contrario, esos y no otros son los dos elementos de la estrategia que ahora mismo se estaría desplegando frente a Corea del Norte.

Una estrategia que, en principio, podría revelarse como exitosa en función de las características del conflicto con Corea del Norte, ya que Estados Unidos y sus aliados cuentan con capacidades militares más que suficientes para asegurar una victoria sobre Corea del Norte en caso de guerra y se encuentran en disposición de ofrecer incentivos a Pyongyang a cambio de que abandone su dinámica de proliferación.

Con todo, para tratar de alcanzar tal éxito será necesario fijar también un límite temporal, que evite las habituales estratagemas norcoreanas para ganar tiempo y envíe una señal a la República Popular Democrática de Corea acerca de la determinación de EE. UU. y sus aliados y su voluntad de terminar rápidamente con el conflicto. Junto a ello, tampoco se debe dejar de dar ciertas garantías a Corea del Norte de que si acepta dar una respuesta positiva a las demandas en relación con sus programas nucleares y de misiles no tiene que temer nuevas existencias en el futuro.

Una estrategia que debería permitir llevar al régimen de Pyongyang a la convicción de que sus programas de armas nucleares, lejos de ser la garantía de su supervivencia, suponen el mayor peligro para la misma, de forma que acepte el abandono - de momento al menos, la congelación y progresivo desmantelamiento- a cambio de ciertas garantías de supervivencia del régimen.

Algo para lo que será imprescindible contar con el concurso de la República Popular China, único actor que aúna unas capacidades y una relación con la República Popular y Democrática de Corea que le permiten ofrecerle garantías de seguridad de la mano de una estrategia de disuasión extendida. Esta posibilidad, más allá de lo que pueda parecer, sería beneficiosa para ambas.

Corea del Norte quedaría liberada del cada vez mayor peso que para su economía supondría mantener su capacidad de disuasión nuclear, especialmente en un contexto de enfrentamiento

\section{La presión} ejercida debe acompañarse de ventajas económicas y de todo tipo lo suficientemente importantes para que Pyongyang tenga la sensación de que también ha salido victorioso de la negociación 
abierto con Estados Unidos y de mejora acelerada de la capacidad de este de lanzar un primer ataque, y el rechazo hacia el mantenimiento del régimen de Pyongyang se reduciría en gran medida.

Por su parte, China, sería la gran beneficiada: por una parte supondría una nueva oportunidad de aparecer como una potencia global responsable, con el consiguiente impacto positivo para ella en términos de imagen tanto a nivel interno como internacional, algo en lo que lleva esforzándose, aunque con altibajos, al menos tres lustros; por otra, y esto es quizás mucho más importante, con la eliminación de las capacidades nucleares de Corea del Norte desaparecería la justificación empleada por Estados Unidos en su última Revisión de la Postura Nuclear para mantener el despliegue de capacidades nucleares dirigidas a objetivos norcoreanos y desplegar capacidades que le permitan interceptar los misiles de la República Popular Democrática de Corea, unas medidas cuyo verdadero objetivo según la percepción de Beijing es limitar la disuasión china (Santoro, 2018); y, por último, se evitaría, al menos por el momento, una Corea reunificada, escenario claramente contrario a las preferencias chinas (Cossa y Glosserman, 2017).

De fracasar esta nueva estrategia, dos serían los escenarios posibles: la aceptación de la modificación del statu quo o el comienzo de un conflicto armado.

\section{Bibliografía}

Abad Quintanal, G. (2012). Las relaciones entre Corea del Norte y Pakistán y el tráfico de tecnología. UNISCI Discussion Papers, (29), 59-65.

Abad Quintanal, G. (2008). La arquitectura de seguridad en Asia-Pacífico. En Anuario Asia Pacífico (pp. 125-133). CIDOB.

Abad Quintanal, G. (2007). Asia: Una región inestable. En Z. Zeraoui (comp.), La Paz y las Regiones del Mundo. Monterrey: Fondo Editorial de Nuevo León.

Alagappa, M. (19 de diciembre 2017). North Korea Must Learn. The Status of a Nuclear Power. Pacific Forum CSIS.

Albert, E. (5 de septiembre de 2017). North Korea's Military Capabilities. Backgrounder. Council of Foreign Relations. Recuperado el 26 de septiembre de 2017 de https://www. cfr.org/backgrounder/north-koreas-military-capabilities

Baker, R. (6 de septiembre 2017). Negociating a Path to Dialogue with North Korea. Strategic Analysis. Stratfor. Recuperado el 26 de septiembre de 2017 de http://worldview.stratfor.com

Bobbitt, P. (21 de septiembre de 2017). A way forward on the Nortk Korea Crisis. Time. Recuperado el 15 de noviembre de 2017 de www.time.com

Byman, D., \& Lind, J. (2010). Pyongyang's Survival Strategy. International Security, (1/Summer), 44-74.

Campbell, C. (21 de agosto de 2017). A war of fiery words, for now, between North Korea and the US. The Brief, 9-10

Campbell, C. (26 de septiembre de 2016). Kim's Last Laugh. Time, 28-31

Choong, W. (30 de agosto de 2017). North Korea: Why It's Time to Double Down on the Double Freeze. The Diplomat. Recuperado el 26 de septiembre de http://thediplomat.com 
Cossa, R. A. (2017a). North Korea: The Storm before the Calm? Pacific Forum CSIS, (29 de noviembre).

Cossa, R. A. (2017b). North Korea: Is a "Grand Bargain” Possible? Pacific Forum CSIS, (20 de abril).

Cossa, R., \& Glosserman, B. (2017). Untying North Korean 'nots'. Pacific Forum CSIS, (14 de febrero).

Cottee, M. (2017). China's Choice: trade with North Korea or with US. IISS (22 de septiembre). Recuperado el 26 de septiembre de 2017 de www.iiss.org

Crystal, S. (2015). Coercive Diplomacy: A Theoretical and Practical Evaluation. Glendon Journal of International Studies, 8(1-2), 1-29

Elleman, M. (2017). The Secret North Korea’s ICBM Success. Survival, 59(5), 25-36.

Elliot, P. (24 de julio de 2017). No good options on North Korea. Time, 10-11.

Ford, G. (2018). The Pyongyang Paradox. Soundings: A journal of politics and culture, (67), 135-146.

Garrido Rebolledo, V. (2017). Incertidumbres nucleares. Política Exterior, (Mayo/Junio), 72-82.

Jongsoo, L. (11 de enero de 2018). An Olympic Window for Korean Nuclear De-escalation. Pacific Forum CSIS.

Jordán Enamorado, J. (2013). Manual de Estudios Estratégicos y Seguridad Internacional. Madrid: Plaza y Valdés.

Knopf, J. W. (2010). The Fourth Wave in Deterrence Research. Contemporary Security Policy, $31(1), 1-33$.

Levy, J. S. (2008) Deterrence and Coercive Diplomacy: The Contributions of Alexander George. Political Psychology, 29(4), 537-552.

Martos, O. J. (2002). Using Conflict Theory. Cambridge: Cambridge University Press.

Minnich, J. M. (2017). North Korea Policy: Changed Regime. Military Review, (noviembrediciembre), 39-53.

Mitchell, A. W. (2 de agosto de 2015). The Case for Deterrence by Denial. The National Interest, (1). Recuperado del 20 de diciembre de 2017 de www.the-american-interest.com

Panda, A. (5 de abril de 2017). North Korean Hackers May Have Seen Secret US-South Korea War Plans. The Diplomat. Recuperado el 26 de septiembre de 2017 de http:// thediplomat.com

Pearlstine, N. (2017). Kim Jong Un: The Threat. The Short List, (5), 96-99.

Santoro, D. (2018). The Point of No Return? China's Choice After the Nuclear Posture Review? Pacific Forum (23 de febrero).

Stanton, J., Lee, S., y Klingner, B. (2017). Getting Tough on North Korea. Foreign Affairs, (mayo-junio), 65-75.

Wilson, W. (2008). The Myth of Nuclear Deterrence. Nonproliferation Review, 15(3), 421-439 